Abstract PS2:23 Table 1 Association between clinics, serology and Anti-C1Q in SLE

\begin{tabular}{|c|c|c|c|c|c|}
\hline $\begin{array}{l}\text { Clinics \& } \\
\text { Serology }\end{array}$ & $\begin{array}{l}\text { Anti-C1Q (+) } \\
(\%)\end{array}$ & $\begin{array}{l}\text { Anti-C1Q (-) } \\
(\%)\end{array}$ & p value & $\begin{array}{l}\text { Odds ratio } \\
\text { (95\% CI) }\end{array}$ & $\begin{array}{l}\text { Adjusted } p \text { value } \\
\text { for age }\end{array}$ \\
\hline Photosensitivity & 90.4 & 16.1 & 0.63 & $1.4(0.3,6.8)$ & 0.01 \\
\hline Malar rash & 56.8 & 56.6 & 0.70 & $0.7(0.1,3.3)$ & 0.06 \\
\hline Discoid rash & 0 & 100 & 0.99 & - & 0.40 \\
\hline Alopecia & 0 & 100 & 0.58 & $1.6(0.2,8.9)$ & 0.5 \\
\hline Oral ulcer & 0 & 100 & 0.64 & $1.6(0.1,13.9)$ & 0 \\
\hline Pleuritis & 0 & 100 & 0.99 & - & 0.16 \\
\hline Pericarditis & 0 & 100 & 0.99 & - & 0.12 \\
\hline Arthritis & 97 & 8.2 & 0.15 & $2.9(0.65,13.6)$ & 0.14 \\
\hline Renal disease & 33.3 & 73.1 & 0.20 & $0.3(0.06,1.8)$ & 0.21 \\
\hline Neurologic disease & 0 & 100 & 0.99 & - & 0.46 \\
\hline Leukopenia & 91.5 & 28.6 & 0.81 & $0.8(0.1,4.4)$ & 0.005 \\
\hline Hemolytic anemia & 0 & 100 & 0.99 & - & 0.52 \\
\hline Thrombocytopenia & 0 & 100 & 0.87 & - & 0.2 \\
\hline Anti-dsDNA & 89.1 & 13.8 & 0.67 & $0.6(0.1,3.7)$ & 0.034 \\
\hline Anti-Smith & 0 & 100 & 0.97 & $1.0(0.1,9.3)$ & 0.73 \\
\hline Low complement & 72.3 & 52.2 & 0.61 & $0.6(0.1,3.6)$ & 0 \\
\hline $\begin{array}{l}\text { Anti-phospholipid } \\
\text { antibody }\end{array}$ & 0 & 100 & 0.40 & $2.4(0.2,20.7)$ & 0 \\
\hline Direct Coombs (+) & 0 & 100 & 0.39 & $0.5(0.1,2.3)$ & 0 \\
\hline
\end{tabular}

patients with other diseases (Sjogrens's syndrome, systemic sclerosis, adult onset Still disease, psoriatic arthritis) and 49 healthy persons (88\% female, mean: 45 years). Anti-C1q was measured by ELISA according to manufacturer's instructions.

Results In SLE group 72 patients had renal, 10 patients had neurologic disease, 25 patients had antiphospholipid syndrome (APS). Anti-dsDNA was positive in 92 patients, anti-Sm was positive in 18 patients, 83 patients had low complement levels. Prevalence of anti-C1q was 5\% (8/150) in patients with SLE and $1 \%(2 / 150)$ in controls $(p=0.88)$. There was no correlation between clinics (renal, neurologic, hematologic, mucocutanous disease, arthritis, serositis, APS) or laboratory findings (Anti-dsDNA, anti-Sm, low complement levels, direct Cooms' test). Within anti-C1q positive group 6 patients had renal disease, 4 had SLEDAI scores 4 or more. None of them had nervous system disease. Six patients had leukopenia, 2 had thrombocytopenia, 5 had mucocutanous disease. Laboratory findings were as follows; 6 patients had anti-dsDNA, 1 had anti-Sm antibody, 6 had low complement levels.

Conclusions Even though there were studies showing the relationship between anti-C1q antibodies and renal disease, it was shown that most of the patients with antibody positivity were Asian ethnicity and younger than 30 years of age. Furthermore anti-C1q antibody was related to disease activation and eliminated within 3 months of treatment. There were also different commercial products available with different cut-off levels. Meta-analysis have shown although anti-C1q antibodies are associated with lupus nephritis, the post-test probabilities are not sufficient to provide certainty of the presence or absence of history of disease.

\section{PS2:24 PLASMATIC AND URINARY ENDOTHELIAL MICROPARTICLES ARE INCREASED IN PATIENTS WITH LUPUS NEPHRITIS}

F Miranda, C Barbati, S Truglia, FR Spinelli, F Ceccarelli, C Alessandri, G Valesini, F Conti. Dipartimento di Medicina Interna e Specialità Mediche, Reumatologia, Sapienza Università di Roma, Italy

\subsection{6/lupus-2018-abstract.72}

Systemic lupus erythematosus(SLE) is a chronic autoimmune disease,characterised by alterations in both the innate and adaptive immune system ultimately leading to the loss of immunologic tolerance and occurrence of autoantibodies against nuclear material. Lupus nephritis is one of the most severe features of SLE determining an increase in morbidity e mortality rates.Renal biopsy still represent a fundamental diagnostic and prognostic tool for LN.Therefore, non-invasive surrogate biomarkers of active LN are urgently needed. Circulating, heterogeneous subcellular microparticles(MPs) are released from cells and platelets constitutively and upon cellular activation or apoptosis.Such MPs may reflect the state of 
their parental cells and tissues, and could serve as markers of pathology. Particularly in SLE, MPs are potential biomarkers and triggers of autoimmunity.Recent studies have demonstrated increased of plasmatic EMPs in patients with SLE active disease and their reduction after treatment.

The aim of this study was to investigate levels of EMPs in a cohort of SLE patients with and without renal involvement compared to healthy controls.

MPs were isolated from plasma and urine and characterised by flow cytometry using AnnessinV (a probe that binds to the exposed phosphatidilserine - PS)and antibodies against surface markers endothelial cells(CD31 +CD41-).

Sixty SLE patients and 29HC were studied. Twenty-eight patients had renal involvement.

The total number of plasmatic MPs was lower in SLE patients than $\mathrm{HC}(\mathrm{p}=0.001)$.

In contrast there was no significant difference EMPs between the two groups. When the patients were divided according to renal involvement, the patients with active-LN(A$\mathrm{LN})$ showed lower plasmatic EMPs in comparison to inactive $\mathrm{LN}(\mathrm{I}-\mathrm{LN}) \quad(\mathrm{p}=0.01)$, while the patients with I-LN had higher EMPs than $\mathrm{HC}(\mathrm{p}=0.002)$. There was no significant difference of total urinaryMPs between SLE patients and HC. UrinaryEMPs were higher in SLE and in LN patients than HC.

The results of the present study show increased EMPs in patients with LN in remission. Circulating-EMPs have been considered as a potential biomarker of endothelial activation and damage in several autoimmune disorders, and higher EMP have been detected in patients with vasculitis and associated with disease activation. According to our results, plasmatic EMPs are higher in inactive-LN patients than in HC. These results may suggest a potential role of EMP as a biomarker of $\mathrm{LN}$.

\section{PS2:25 ANALYSIS OF THE POLYAMINE METABOLOME IN THE PLASMA OF PATIENTS WITH SYSTEMIC LUPUS ERYTHEMATOSUS AND FEVER}

C Suh, W Baek, S Lee, H Kim, J Jung. Ajou University School of Medicine, Suwon, South Korea

\subsection{6/upus-2018-abstract.73}

Introduction Systemic lupus erythematosus (SLE) is a systemic autoimmune disease with various clinical manifestations and serologic markers. In this study, we analysed nine polyamine profiles of plasma from patients with SLE and healthy controls, and the relationship between the polyamine profiles and disease activity.

Methods The alterations of the polyamine metabolome in the plasma of 44 patients with SLE and fever were investigated using gas chromatography mass spectrometry in selected ion monitoring mode using N-ethoxycarbonyl/N-pentafluoropropionyl derivatives, and compared with those of 43 healthy controls.

Results Patients with SLE and healthy controls showed differences in five of nine polyamine metabolome profiles. Among five polyamines changed levels, four polyamines, namely N1acetylcadaverine, spermidine, N1-acetylspermidine, and spermine, were dramatically decreased. However, the level of cadaverine was increased in patients with SLE. In the partial correlation with polyamine profiles and disease activity markers of SLE, several disease activity markers and nutritional markers were correlated with cadaverine, spermidine, and N8-acetylspermidine.

Conclusion Thus, our results provide a comprehensive understanding of relationship between that polyamine metabolomes and disease activity markers in patients with SLE and fever.

\section{PS2:26 SLE PATIENTS WITH SECONDARY SJÖGREN'S SYNDROME ARE CHARACTERISED BY TYPICAL AUTOANTIBODIES AND A PRO-INFLAMMATORY STATE}

${ }^{1} \mathrm{M}$ Kvarnström, ${ }^{2} \mathrm{G}$ Ruacho, ${ }^{1} \mathrm{~J}$ Gustafsson, ${ }^{1} \mathrm{~A}$ Zickert, ${ }^{1} \mathrm{~V}$ Oke, ${ }^{3} \mathrm{~J}$ Rönnelid, ${ }^{4} \mathrm{~K}$ Elvin, ${ }^{1}$ I Gunnarsson, 'E Svenungsson. 'Unit of Rheumatology, Department of Medicine Solna, Karolinska Institutet, Karolinska University Hospital, Stockholm, Sweden; ${ }^{2}$ Centre for Clinical Research Sörmland, Uppsala University, Uppsala, Sweden; ${ }^{3}$ Department of Immunology, Genetics and Pathology, Uppsala University, Uppsala, Sweden; ${ }^{4}$ Unit of Clinical Immunology, Department of Clinical Immunology and Transfusion Medicine, Karolinska Institutet, Karolin, Stockholm, Sweden

\subsection{6/lupus-2018-abstract.74}

Background Sjögren's syndrome occurs in isolation (primary Sjögren's syndrome, pSS), but it is also often secondary (sSS) to, and sometimes difficult to delineate from systemic lupus erythematosus (SLE). Consequently there is a need to investigate similarities and differences between SLE patients with (SLE-sSS) and without sSS (SLE-noSS).

Objective To investigate the occurrence of sSS in a large cohort of SLE patients and to explore clinical and laboratory characteristics associated with SLE-sSS as compared to SLEnoSS and controls.

Methods We included 504 consecutive SLE patients and 322 population controls, matched for age and gender. All patients fulfilled the 1982 revised ACR criteria for SLE. SLE-sSS was defined according to the American-European consensus criteria (AECC). Subjective and objective quantifications of sicca symptoms were recorded. All underwent a thorough clinical investigation. SLE-associated autoantibodies, (ANA screening by BioPlex 2200 system, Bio-Rad) and Rheumatoid factor (Rf, Phadia Immunocap 250) were determined, Routine laboratory workup and a panel of cytokines (MSD 30-plex cytokine assays, performed on samples from 433 consecutive SLE patients and 319 controls) were measured.

Results SLE-sSS, occurred in 23\% of the SLE patients. Compared to SLE-noSS the SLE-sSS group was older, both at inclusion (55 vs 43 years, $\mathrm{p}<0.0001)$ and at disease onset $(40$ vs 32 yrs $\mathrm{p}<0.0001$ ), and more enriched in females (96 vs $83 \%, \mathrm{p}=0.0007)$, Leucopenia (57 vs $45 \%, \mathrm{p}=0.02)$ and peripheral neuropathy ( 15 vs $7 \%, \mathrm{p}=0.01)$ were more common and nephritis less common ( 32 vs $43 \%, p=0.03$ ). Higher levels of total IgG, positivity for anti-SSA/Ro52, anti-SSA/ Ro60, anti-SSB antibodies, Rf IgM and Rf IgA characterised the SLE-sSS group. 19/20 levels of detected cytokines were higher in SLE than in controls. 6/20 cytokines (TNF-a, IL-6, MCP-4, MIP-1 $\beta$, IL12/IL-23p40 and IP-10) were upregulated in SLE-sSS vs SLE-noSS (see table for figures).

Conclusion Frequency of SLE-sSS increases with age and affects roughly $1 / 4$ of SLE patients. Nephritis was less common while leucopenia and peripheral neuropathy were more common. We report higher levels of six pro-inflammatory. These findings demonstrate that, though often regarded as a milder version of SLE, patients with SLE-sSS are characterised by a state of chronic systemic inflammation. 